\title{
¿Qué sabe el alumnado de secundaria sobre la Unión Europea? Implicaciones para el aprendizaje
} What do secondary school pupils know about the European Union? Implications for learning

\author{
Santana-Vega, Lidia E. \& Feliciano-García, Luis
}

Universidad de La Laguna (España)

\begin{abstract}
The development of a European Dimension in Education is an objective linked to the construction of European identity and the exercise of active, responsible and critical citizenship. The paper analyzes the knowledge of Spanish Compulsory Secondary School pupils on topics regarding the European Union. The research involved 705 pupils from five secondary education centres in four Spanish regions. Twenty four item test was designed to examine the pupils' knowledge about: a) the European institutions, b) history of European cooperation, c) European citizenship, d) Europe in daily life, e) social and cultural identity and f) human rights and responsibilities. The results show that: a) $80.3 \%$ of the pupils only answered 14 items or less correctly; b) there were significant differences among pupils according to the year of study and the region, but a small size effect; c) pupils lack knowledge regarding "Europe in everyday life”, "European Citizenship", "History of European cooperation" and "The European institutions". There is a discussion of the need to define a cross-curricular approach.
\end{abstract}

\section{Keywords:}

Citizenship education; Secondary education; Educational testing; Student evaluation; European dimension in education

\begin{abstract}
Resumen
El desarrollo de la Dimensión Europea en la Educación es un objetivo vinculado a la construcción de la identidad europea y al ejercicio de una ciudadanía activa crítica y responsable. El artículo analiza el conocimiento de los estudiantes de Educación Secundaria Obligatoria sobre la Unión Europea. En el estudio participaron 705 alumnos de cinco centros de educación secundaria obligatoria de cuatro comunidades autónomas españolas. Se diseñó una prueba de veinticuatro ítems sobre: a) las instituciones europeas, b) la historia de la cooperación europea, c) la ciudadanía Europea, d) Europa en la vida cotidiana, e) la identidad social y cultural, y f) derechos humanos y responsabilidades. Los resultados muestran que: a) el $\mathbf{8 0 . 3 \%}$ de los alumnos contestaron correctamente catorce o menos ítems; b) existen diferencias significativas entre los alumnos en función del curso y de la comunidad autónoma, pero el tamaño del efectos es pequeño; c) los alumnos carecen de conocimientos sobre "Europa en la vida cotidiana”, "la Ciudadanía Europea”, "la Historia de la cooperación europea” y "las Instituciones europeas”. Se discute la necesidad de diseñar una propuesta curricular transversal.
\end{abstract}

\section{Reception Date} 2017 January 17

Approval Date 2017 December 20

Publication Date: 2017 November 21

\section{Palabras clave:}

Educación para la ciudadanía; Educación secundaria; Test de conocimiento; Evaluación del alumnado; Dimensión Europea en la educación.

El aprendizaje de la ciudadanía debe tener lugar tanto en el contexto de la educación formal como no formal; como dice un viejo y sabio proverbio africano "se necesita toda la tribu para educar a un niño". La educación para la ciudadanía es una prioridad urgente en un mundo globalizado, ya que es necesario revertir la deseducación democrática de la ciudadanía. Según Greppi (2012) muchas sociedades democráticas están atrapadas en un círculo perverso de "deseducación democrática". El círculo se cierra cuando la 
cantidad de ciudadanos mal educados supera en número a los ciudadanos bien educados. Las mayorías mal educadas terminan votando en contra de sus propios intereses, en otras palabras, eligen líderes que saben muy bien cómo fomentar la mala educación sistemática de la ciudadanía para alcanzar sus propios fines. Bovero (2002) plantea las siguientes preguntas: ¿cómo puede restablecerse el círculo virtuoso entre democracia y progreso?; ¿cómo se pueden reparar las estructuras de mediación democrática necesarias para una participación activa de los ciudadanos en los procesos de formación de la opinión pública y la voluntad colectiva? Quizá fomentando el "espíritu de iniciativa" los ciudadanos en la era de la globalización podrán responder activamente y de manera creativa y estratégica a los desafíos de la ciudadanía global en el siglo XXI.

La complejidad de la sociedad contemporánea requiere la articulación de buenos planes de estudio para fomentar entre el alumnado la educación ciudadana, el espíritu de iniciativa y de autonomía, y la capacidad de tomar decisiones prudentes e informadas. El discurso sobre la educación para la ciudadanía es importante, la forma en que ésta se representa determina quiénes serán incluidos o excluidos como buenos ciudadanos (Myers \& Zaman, 2009). La consolidación de la ciudadanía europea requiere que los ciudadanos de la Unión Europea (UE): a) se consideren participantes en la construcción de la UE, b) conozcan sus derechos y deberes, y c) vean a la UE como algo más que un conjunto de instituciones económicas y administrativas (Comisión Europea, 2011; Hernández Rodríguez, 2008).

El proceso de integración europea ha sido gestionado por instituciones percibidas por los ciudadanos europeos como alejadas de su vida cotidiana. Una parte importante de los ciudadanos europeos considera que la UE es una entidad política distante (Comisión Europea, 2011). Los resultados de los informes más recientes de la Comisión Europea muestran que los ciudadanos de la UE no asumen plenamente la ciudadanía europea. Según el Flash Eurobarómetro 365 (Comisión
Europea, 2013), solo el $46 \%$ de los encuestados estaba familiarizado con el término "ciudadano europeo" y entendía su significado. El Eurobarómetro estándar 77 (Comisión Europea, 2012) señala que solo el $19 \%$ de la población de la UE sentía que eran "ciudadanos europeos", el $42 \%$ se sentía "en cierta medida ciudadanos europeos" y el 38\% "no se sentían ciudadanos europeos". El Eurobarómetro estándar 81 (Comisión Europea, 2014) muestra un ligero cambio en estos porcentajes, que son del 26\%, 39\% y $34 \%$, respectivamente.

El programa "Europa con los ciudadanos (2014-2020)" (Consejo de la Unión Europea, 2014) es un ejemplo de la línea de acción principal de la UE, cuyos objetivos son: a) ofrecer a los ciudadanos la oportunidad de interactuar y participar en la construcción de una Europa cada vez más cercana, b) forjar una identidad europea basada en los valores comunes, la historia y la cultura, c) mejorar la comprensión y el respeto mutuo entre los ciudadanos europeos, d) fomentar el sentido de pertenencia a la Unión Europea entre sus ciudadanos, e) mejorar la comprensión mutua entre los ciudadanos europeos respetando $\mathrm{y}$ promoviendo la diversidad cultural $\mathrm{y}$ lingüística, y f) fomentar la participación democrática de los ciudadanos en la UE, desarrollando la comprensión del proceso de elaboración de las políticas de la UE y promoviendo oportunidades para el compromiso social e intercultural, y el voluntariado en la UE (Consejo de la Unión Europea, 2014).

Este trabajo se basa en un proyecto financiado por la Comisión Europea destinado a promover la construcción de la ciudadanía europea y el espíritu de iniciativa a través de un programa educativo para la Educación Secundaria (Bombardelli y Santana, 2014). Los objetivos de la investigación son: 1) analizar el conocimiento de los alumnos españoles de Educación Secundaria sobre temas relativos a la UE y al ejercicio de la ciudadanía europea, 2) analizar si existen diferencias en el conocimiento de los alumnos sobre la UE según la región, curso o sexo; 3) examinar las cuestiones relativas a la UE que 
el alumnado necesita aprender más profusamente.

\section{Antecedentes}

El desarrollo de la ciudadanía es esencial para afrontar los desafíos del ámbito nacional e internacional (del Río, 2009). Frente a la narrativa nacional dominante, es necesario preparar a los jóvenes para la ciudadanía en un mundo globalizado, dado que se implican cada vez más en múltiples asociaciones cívicas, adoptan creencias y llevan a cabo acciones que van más allá de las fronteras nacionales (Myers y Zaman, 2009; UNESCO, 2015).

El Consejo de Europa ha enfatizado la importancia de la educación para el ejercicio de una ciudadanía "activa", "comprometida" y "participativa" (Marshall, 2009). La educación para el desarrollo de una ciudadanía plena ayudará a garantizar la estabilidad y la legitimidad de la UE y a responder al incremento de las formas no democráticas de activismo político (Milana, 2008). El aprendizaje de los derechos y deberes de los ciudadanos europeos, los valores democráticos, los derechos humanos y los canales de participación en una sociedad democrática promueven cambios en el comportamiento de los jóvenes. Este aprendizaje es necesario para que los jóvenes puedan comportarse como ciudadanos responsables y desempeñar un papel activo en la construcción de la UE (EURYDICE, 2005). Por tanto, la educación es fundamental para aumentar la implicación y la participación de los ciudadanos europeos (EACEA 2012). Según Naval, Print y Veldhuis (2002) la ciudadanía no solo se considera como un estatus legal, sino también como una competencia, cuya adquisición debe desarrollarse a través de la educación en las escuelas y en otros entornos. Las competencias sociales y cívicas son parte de las ocho competencias clave para la formación de ciudadanos europeos; su adquisición es esencial para la realización personal y el compromiso con la ciudadanía activa (Halász y Michel, 2011).

El desarrollo de una dimensión europea en la educación es un objetivo relacionado con la construcción de la identidad europea y el ejercicio de la ciudadanía activa. La Declaración de París sobre la promoción de la ciudadanía y los valores comunes de libertad, tolerancia y no discriminación a través de la educación (EACEA, 2016) enfatiza que la dimensión europea en educación apoya el desarrollo de la ciudadanía europea a través de: a) la información y el trabajo en común, b) el aprendizaje de ideas y culturas de otros países, c) la socialización a través de intercambios, d) una mayor comprensión de la "realidad" de la integración europea, y e) la transmisión de valores que sustentan el sentimiento de pertenencia común.

La idea es "Entender Europa, Sentir Europa y Participar en Europa" para enfrentar los desafíos de desinformación que siente una parte de la ciudadanía europea (Hernández Rodríguez, 2008). Una pregunta clave para la dimensión europea en la educación es: ¿cómo debería ser la educación de los ciudadanos europeos? Muchos proyectos y programas han intentado responder a esta pregunta; muchos de ellos han creado recursos de gran interés para mejorar la calidad de la educación para la ciudadanía europea.

Diversas investigaciones realizadas con jóvenes para estudiar su sentido de pertenencia a la UE, su conocimiento del significado de la ciudadanía europea y su deseo de participar en la construcción de la UE (Jamieson 2007; Mahendran y Cook, 2007; Huyst, 2009; Agidarg, Huyst, y Van Houte, 2012; Horvath y Paolini, 2013; Sloam, 2013; EACEA, 2013; Kuhn, 2016) concluyen que: a) los jóvenes no rechazan los objetivos de la UE y consideran que la pertenencia de su país a la UE es beneficiosa; b) tienen una visión más positiva de la ciudadanía europea y del proceso de integración europea que otros grupos de edad; c) quieren tener una mayor voz y más oportunidades para votar; d) están interesados en ser parte activa del proceso de integración; e) demandan más información sobre las ventajas de ser miembro de la UE; f) saben menos sobre la UE que los grupos de mayor edad; g) el estatus socioeconómico de los padres influye en las interacciones transnacionales de los jóvenes en los países 
europeos; h) los jóvenes que viven en economías más ricas son más transnacionales. Las conclusiones del Estudio Internacional de Educación Cívica y Ciudadanía (ICCS) 2009 (Kerr, Sturman, Schulz \& Burge, 2010), realizado en 24 países europeos, indican que el conocimiento sobre la UE del alumnado de Educación Secundaria es relativamente bueno, pero necesita ser mejorado.

La Comisión de Cultura y Educación del Parlamento Europeo (2016) señala: 1) la creciente importancia de una dimensión europea en la educación en las diferentes disciplinas, niveles y formas de educación; 2) la necesidad de que la UE sea más visible y se integre mejor en los materiales de enseñanza y las actividades extracurriculares, dado su impacto en la vida cotidiana de sus ciudadanos; 3) el valor sustancial que los contenidos explícitamente relacionado con la UE pueden agregar a los planes de estudios escolares y al desarrollo personal y el crecimiento de los estudiantes. La educación para la ciudadanía europea y la adquisición de competencias sociales y cívicas deben lograrse a través de la enseñanza en el aula, las actividades participativas en la escuela y en las comunidades locales. El aprendizaje de estas competencias es una oportunidad para: a) experimentar los valores y principios de la participación pública, y b) evitar que se pierda la valiosa contribución de los jóvenes.

\section{Método}

Dada la ausencia de estudios previos en España sobre los conocimientos que el alumnado de Educación Secundaria Obligatoria tiene del proceso de integración europea y la ciudadanía europea, esta investigación es exploratoria. Se llevó a cabo un estudio de encuesta en el que se aplicó una prueba de conocimiento diseñada ad hoc para alumnado de Educación Secundaria Obligatoria.

\section{Muestra}

El proyecto se desarrolló en centros públicos de educación secundaria de cuatro Comunidades Autónomas de España (Islas Canarias, Andalucía, Aragón y Castilla-La Mancha). Estos centros decidieron colaborar en el proyecto por dos motivos: 1) su interés por mejorar el conocimiento de su alumnado sobre la ciudadanía europea y 2) su deseo de promover las competencias sociales y cívicas del alumnado. Es una muestra de conveniencia, no representativa de las diferentes comunidades autónomas en las que se encuentran los centros participantes. 705 alumnos españoles y 40 profesores de Educación Secundaria Obligatoria (ESO) de cinco centros participaron en el proyecto (Tabla 1). El 51.2\% de los alumnos eran varones y el $48.8 \%$ mujeres. Las edades del alumnado estaban comprendidas entre los 12$13(44,7 \%)$ y $14-15$ (55,3\%) años de edad (media: 13,72, desviación estándar: 0,9369); el $53.5 \%$ de los alumnos estaban en segundo curso y $46.5 \%$ en tercero.

Tabla 1. Datos de los centros de Educación Secundaria Obligatoria Españoles

\begin{tabular}{cccl}
\hline Comunidad Autónoma & Alumnos & Profesores & \multicolumn{1}{c}{ Materias escolares implicadas } \\
Andalucía & 157 & 10 & $\begin{array}{l}\text { Lengua Española y Literatura, Geografía, Historia, Tecnología, } \\
\text { Arte, Matemáticas }\end{array}$ \\
Islas Canarias & 190 & 14 & $\begin{array}{l}\text { Inglés, Lengua Española y Literatura, Matemáticas, Francés, } \\
\text { Ciencias Sociales, Tecnología }\end{array}$ \\
Aragón & 214 & 8 & $\begin{array}{l}\text { Ciencias Sociales, Ciencias, Tecnología, Inglés, Tutoría } \\
\text { Castilla-La Mancha }\end{array} 199$ \\
\hline
\end{tabular}

\section{Instrumentos}

El proyecto incluyó una prueba diseñada para examinar el conocimiento del alumnado sobre el proceso de integración europea y la ciudadanía europea. En el diseño de la prueba se tuvieron en cuenta: a) los contenidos relevantes sobre el proceso de integración europea y ciudadanía europea, y b) la 
información ofrecida en los libros de texto de Educación Secundaria sobre estos temas.

Se analizaron sitios web institucionales, sitios web de enseñanza, blogs y libros de texto de Educación Secundaria para seleccionar el contenido del test. Sobre la base de este análisis, se eligieron los siguientes contenidos: 1) UE: concepto y Estados miembros. 2) Orígenes de la UE: antecedentes y objetivos de su creación, y los símbolos de la UE. 3) Valores fundadores de la UE: libertad, democracia, respeto por los derechos humanos, estado de derecho. 4) Instituciones de la UE. 5) Ciudadanía europea: creación, significado, objetivos, relación entre la ciudadanía europea y la nacionalidad. 6) Derechos europeos de ciudadanía. 7) La moneda europea: los objetivos de la introducción del euro, la zona del euro, la financiación de la UE, las becas y subsidios de la UE. Estos temas se usaron en el diseño de un primer borrador de la prueba que tenía 80 ítems. El primer borrador fue revisado por profesores especializados en la UE de las cinco universidades participantes. La prueba también fue revisada por maestros de educación secundaria que colaboraron en el proyecto.

La prueba se analizó y reestructuró en el transcurso de varias reuniones de los socios del proyecto y los docentes colaboradores de educación secundaria. Las siguientes decisiones se adoptaron después del proceso de discusión: a) reducir el número de preguntas, b) introducir nuevos ítems, c) cambiar algunas frases $\mathrm{y}$ alternativas de respuesta. El objetivo de las sucesivas mejoras del test era garantizar que: 1) contenía cuestiones básicas, según los expertos, sobre la UE y la ciudadanía europea, y 2) se adaptaba a la población destinataria del estudio. Los participantes en el proceso de revisión estuvieron totalmente de acuerdo con el contenido de la prueba. La única discrepancia se produjo en el caso de los ítems sobre la economía de la UE, como sucedió en el estudio de Amadeo, Torney-Purta, Lehmann, Husfeldt y Nikolova (2002). Algunos docentes consideraron que el tema no era apropiado para los alumnos de entre 12 y 15 años. En consecuencia, los ítems sobre economía fueron eliminados.

El resultado del proceso de revisión fue un test de 24 ítems. Cada ítem tenía cuatro respuestas posibles de las cuales solo una era correcta. La puntuación final del test de conocimiento varió entre 0 y 24 puntos. Los ítems en la versión final de la prueba versaban sobre: 1) derechos humanos y responsabilidades, 2) identidad social y cultural, 3) Europa en la vida cotidiana, 4) ciudadanía europea: 5) historia de la cooperación europea y 6) las instituciones europeas.

La correspondencia de los ítems con los temas del test se muestra en la Tabla 2: el $12.5 \%$ de los ítems examina contenidos sobre los Derechos Humanos y Responsabilidades (por ejemplo, ¿Qué establece la Carta de los Derechos Fundamentales de la UE?); el 12.5\% analiza contenidos sobre identidad social y cultural (por ejemplo, ¿qué significa "tener un prejuicio"); el 20,8\% examina contenidos sobre Europa en la vida cotidiana (por ejemplo, ¿qué ocurre con la soberanía de los países que se unen a la UE?); el $25 \%$ analiza contenidos sobre ciudadanía europea (por ejemplo, ¿en qué situaciones respetamos los bienes públicos?); el $12.5 \%$ analiza contenidos sobre la historia de la cooperación europea (por ejemplo, ¿cuál es el objetivo del proceso de integración europea?); el $16,7 \%$ examina contenidos sobre las instituciones europeas (por ejemplo, ¿cómo se eligen los miembros del Parlamento Europeo?).

Tabla 2. Correspondencia de los ítems con los temas del test de conocimiento

\begin{tabular}{lcc}
\hline \multicolumn{1}{c}{ Temas del Test } & Ítems del Test & Total \\
1. Derechos humanos y responsabilidades & $1,2,3$ & 3 \\
2. Identidad social y cultural & $4,5,6$ & 3 \\
3. Europa en la vida cotidiana & $7,8,9,10,11$ & 5 \\
4. Ciudadanía europea & $12,13,14,15,16,17$ & 6 \\
5. Historia de la cooperación europea & $18,19,20$ & 3 \\
6. Las instituciones europeas & $21,22,23,24$ & 4 \\
\hline
\end{tabular}


El índice de fiabilidad de la prueba, calculado mediante el coeficiente de las dos mitades de Guttman, fue bajo $(0,61)$. El índice de discriminación de la prueba es 0.83 . Este valor muestra que la prueba diferencia a los alumnos con niveles altos y bajos de conocimiento sobre la UE. El análisis del índice de discriminación para cada ítem muestra que doce ítems (50\%) tienen un índice excelente, cinco (21\%) tienen un índice bueno, tres (12.5\%) un índice regular y cuatro (16.5\%) un índice pobre (ver Tabla 3). Los ítems con el índice de discriminación más bajo pertenecen a los temas: instituciones europeas (4 ítems), ciudadanía europea (2 ítems) y Europa en la vida diaria (1 ítem).

\section{Procedimiento}

La investigadora principal del proyecto en España se reunió con los equipos directivos de los centros participantes, explicó los aspectos más relevantes del proyecto en una sesión informativa y los remitió al sitio web del proyecto para obtener más información. El director de cada centro aceptó la solicitud de informar al personal docente sobre el proyecto y solicitar la colaboración del profesorado de los diferentes departamentos educativos. Cada centro eligió un coordinador de proyecto. Este coordinador fue elegido entre el profesorado que habían decidido participar en el proyecto. El investigador principal explicó las características y dimensiones de la prueba de conocimiento a los profesores de las escuelas participantes. La prueba fue aplicada en el aula por el profesorado que colaboró en el proyecto.

El análisis de los datos del test de conocimiento se realizó utilizando el programa SPSS.21 e incluyó: a) coeficiente de fiabilidad para la prueba mediante el método de dos mitades de Guttman; b) índice de dificultad y discriminación de cada ítem; c) estadísticos descriptivos para la distribución de las puntuaciones totales en el tests; d) Prueba ANOVA y T de Student para muestras independientes para comprobar la existencia de diferencias significativas entre las puntuaciones medias del test según sexo, comunidad autónoma y curso; e) análisis de efecto de tamaño (d de Cohen y parcial $\eta^{2}$ ); f) porcentaje de respuestas correctas para cada elemento, g) estadísticos descriptivos del porcentaje de respuestas correctas en el test por temas; h) ANOVA de medidas repetidas para comprobar la existencia de diferencias significativas entre los porcentajes medios de respuestas correctas en los temas del test y el ajuste de Bonferroni para comparaciones múltiples por pares entre las medias marginales del efecto principal; se usó $\eta^{2}$ parcial como índice de tamaño del efecto.

El Índice de Dificultad muestra la proporción de respuestas correctas del 25\% de los participantes con puntuaciones más altas en el test, y el 25\% inferior de los participantes con las puntuaciones más bajas. El Índice de Dificultad es el promedio del $25 \%$ superior y el $25 \%$ inferior de los participantes. Cuanto mayor sea el valor del índice, menor será la dificultad, cuanto mayor sea la dificultad de un ítem, menor será su índice.

El Índice de Discriminación es la diferencia entre dos proporciones, las respuestas correctas del grupo superior menos las respuestas correctas del grupo inferior. Cuanto mayor es la diferencia entre el número de respuestas correctas entre los grupos superior e inferior, más discriminatorio es el ítem; esto ayuda a ubicar a un participante en el grupo superior o inferior (Morales, 2012). Los criterios de Ebel y Frisbie (1986) y Morales (2012) se tuvieron en cuenta para evaluar los índices de dificultad y discriminación de los ítems.

\section{Resultados}

La distribución de las puntaciones del alunado en el test de conocimiento muestra que el $53 \%$ de los sujetos contestó correctamente solo entre 10-14 ítems (ver Figura 1); el 27.3\% tuvo una puntuación baja en la prueba, contestando correctamente 9 preguntas o menos. Solo el $19.7 \%$ de los alumnos respondieron correctamente 15 o más preguntas. Según la distribución de las puntuaciones en el test, el nivel de conocimiento del alumnado sobre el proceso de integración europea y la ciudadanía europea es medio bajo. 


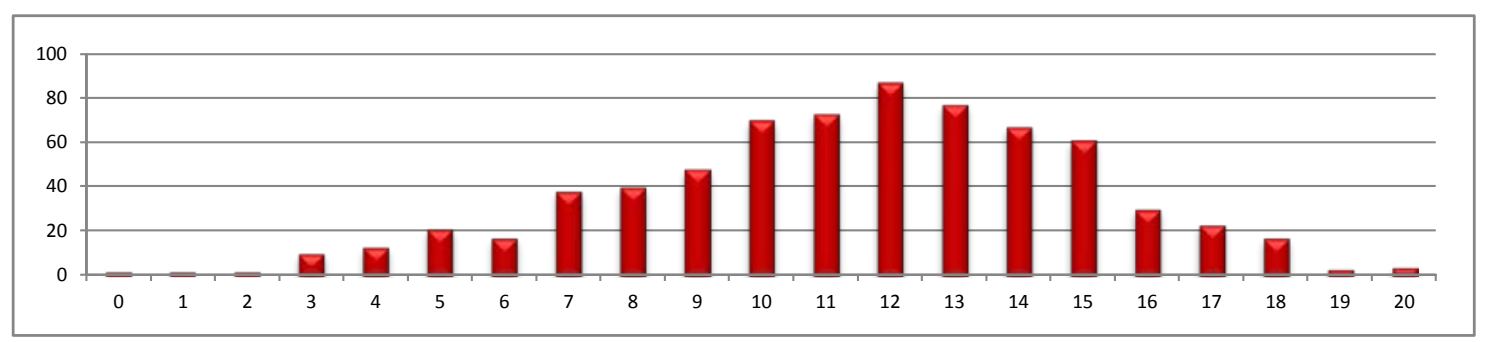

Figura 1. Distribución de las puntuaciones del test de conocimiento

Media: 11.38, Mediana: 12, Moda: 12, Desviación Típica: 3.59 Asimetría: -0.353

La tasa de dificultad general del test es de 0.47. Esta tasa indica que el test tiene un nivel medio-alto de dificultad (Ebel y Frisbie, 1986; Morales, 2012). Los ítems del test tienen índices de dificultad dentro del rango de 0.79 y 0.14. Cuatro preguntas tienen una tasa de dificultad alta (16,7\%), once tienen una tasa medio-alta $(45,83 \%)$, ocho tienen una tasa de dificultad media $(33,3 \%)$ y solo un elemento tiene una tasa de dificultad medio-baja (4,2\%). (Tabla 3).

Tabla 3. Índice de dificultad y de discriminación de los ítems del test de conocimiento

\begin{tabular}{|c|c|c|c|c|c|}
\hline \multirow{2}{*}{ Tema } & \multirow{2}{*}{ Ítems } & \multicolumn{2}{|c|}{ İndice de Discriminación } & \multicolumn{2}{|c|}{ Índice de Dificultad } \\
\hline & & Valor & Discriminación & Valor & Dificultad \\
\hline \multirow{3}{*}{1} & $\begin{array}{l}\text { 1. ¿Qué países de la UE han adoptado la Declaración } \\
\text { Universal de los Derechos Humanos? }\end{array}$ & 0.35 & Bueno & 0.42 & Medio-Alto \\
\hline & $\begin{array}{l}\text { 2. ¿Qué establece la Carta de los Derechos Fundamentales } \\
\text { de la UE? }\end{array}$ & 0.41 & Excelente & 0.69 & Medio \\
\hline & $\begin{array}{l}\text { 3. ¿Cuál de las siguientes situaciones es un acto de } \\
\text { "discriminación"? }\end{array}$ & 0.31 & Bueno & 0.79 & Medio-Bajo \\
\hline \multirow{3}{*}{2} & 4. ¿Qué significa "tener un prejuicio"? & 0.34 & Bueno & 0.36 & Medio-Alto \\
\hline & 5. ¿Cuál es la utilidad de aprender un idioma extranjero? & 0.52 & Excelente & 0.64 & Medio \\
\hline & $\begin{array}{l}\text { 6. ¿Cuál es aproximadamente el porcentaje de inmigrantes } \\
\text { en España? }\end{array}$ & 0.47 & Excelente & 0.69 & Medio \\
\hline \multirow{5}{*}{3} & 7. ¿Cuántos países forman la UE? & 0.50 & Excelente & 0.59 & Medio \\
\hline & 8. Aproximadamente, ¿cuántos residentes hay en la UE? & 0.29 & Regular & 0.43 & Medio-Alto \\
\hline & 9. ¿Qué significa la "libre circulación de bienes"? & 0.51 & Excelente & 0.41 & Medio-Alto \\
\hline & $\begin{array}{l}\text { 10. ¿Qué sucede con la soberanía de los países que se unen } \\
\text { a la UE? }\end{array}$ & 0.48 & Excelente & 0.46 & Medio-Alto \\
\hline & $\begin{array}{l}\text { 11. ¿Dónde puedes encontrar información sobre el proceso } \\
\text { de integración europea? }\end{array}$ & 0.49 & Excelente & 0.51 & Medio \\
\hline \multirow{6}{*}{4} & 12. ¿Qué sucede cuando obtienes la ciudadanía europea? & 0.19 & Pobre & 0.31 & Medio-Alto \\
\hline & $\begin{array}{l}\text { 13. ¿Qué sucede cuando tienes un problema de salud y } \\
\text { trabajas en otro país perteneciente a la UE? }\end{array}$ & 0.60 & Excelente & 0.54 & Medio \\
\hline & $\begin{array}{l}\text { 14. ¿Dónde puede encontrar normas de la UE en la vida } \\
\text { cotidiana? }\end{array}$ & 0.23 & Regular & 0.29 & Alto \\
\hline & $\begin{array}{l}\text { 15. ¿En qué situaciones respetamos la propiedad y los } \\
\text { bienes públicos? }\end{array}$ & 0.54 & Excelente & 0.66 & Medio \\
\hline & 16. ¿Qué es la UE en el mundo? & 0.49 & Excelente & 0.41 & Medio-Alto \\
\hline & $\begin{array}{l}\text { 17. ¿Cuál de los siguientes países requiere un pasaporte } \\
\text { para entrar en él? }\end{array}$ & 0.40 & Excelente & 0.42 & Medio-Alto \\
\hline \multirow{3}{*}{5} & $\begin{array}{l}\text { 18. ¿Cuál es el objetivo del proceso de integración } \\
\text { europea? }\end{array}$ & 0.59 & Excelente & 0.64 & Medio \\
\hline & 19. ¿Cuándo nació la comunidad europea? & 0.38 & Bueno & 0.36 & Medio-Alto \\
\hline & 20. ¿Qué es el Tratado de Lisboa? & 0.36 & Bueno & 0.38 & Medio-Alto \\
\hline \multirow{4}{*}{6} & $\begin{array}{l}\text { 21. ¿Cómo se elige a los miembros del Parlamento } \\
\text { Europeo? }\end{array}$ & 0.13 & Pobre & 0.21 & Alto \\
\hline & $\begin{array}{l}\text { 22. ¿Quién es miembro del Parlamento Europeo de tu } \\
\text { país? }\end{array}$ & 0.09 & Pobre & 0.14 & Alto \\
\hline & $\begin{array}{l}\text { 23. ¿Cuántos países de la UE usan el euro como moneda } \\
\text { oficial? }\end{array}$ & 0.10 & Pobre & 0.18 & Alto \\
\hline & 24. ¿Quién es el presidente de la Comisión Europea? & 0.23 & Regular & 0.33 & Medio-Alto \\
\hline
\end{tabular}


Los ítems donde el porcentaje de respuestas incorrectas fue mayor del 50\% pertenecían a los siguientes temas: las instituciones europeas (items 21, 22, 23, 24), ciudadanía europea $(12,14,16,17)$, Europa en la vida cotidiana (ítems 8, 9, 10 y 11), historia de la cooperación europea (ítems 19 y 20), derechos humanos y responsabilidades (ítem 1), identidad social y cultural (ítem 4).

El ANOVA en función de la comunidad autónoma mostró diferencias significativas entre el porcentaje promedio de las respuestas correctas de los grupos ( $\mathrm{F}=4.980$, gl: $3, \mathrm{p}$ $<0.002)$. Sin embargo, el coeficiente $\eta^{2}$ parcial (0.021) indicaun tamaño del efecto pequeño. Los resultados de las comparaciones múltiples post hoc utilizando la prueba de Tukey muestran que el alumnado de Andalucía tenía un porcentaje promedio de respuestas correctas significativamente más alto que el alumnado de las Islas Canarias y Aragón (Tabla 4).

Tabla 4. Contraste de medias según Comunidad Autónoma

\begin{tabular}{|c|c|c|c|c|c|c|}
\hline \multirow{2}{*}{ Región } & \multirow{2}{*}{ Media } & \multirow{2}{*}{ DT } & \multirow{2}{*}{$\begin{array}{l}\text { Diferencia } \\
\text { Medias }\end{array}$} & \multicolumn{2}{|c|}{ Intervalos de confianza } & \multirow{2}{*}{ Sig. } \\
\hline & & & & Límite inferior & Límite Superior & \\
\hline $\begin{array}{l}\text { Andalucía } \\
\text { Canarias }\end{array}$ & $\begin{array}{l}50.31 \\
45.37\end{array}$ & $\begin{array}{l}13.91 \\
15.76\end{array}$ & 4.9421 & .6136 & 9.2707 & 0.018 \\
\hline $\begin{array}{l}\text { Andalucía } \\
\text { Aragón }\end{array}$ & $\begin{array}{l}50.31 \\
45.27\end{array}$ & $\begin{array}{l}13.91 \\
15.04 \\
\end{array}$ & 5.0464 & .9379 & 9.1550 & 0.009 \\
\hline
\end{tabular}

El contraste de medias según el curso muestra que el alumnado de tercero obtuvo una puntuación media significativamente más alta en el test que el alumnado de segundo (Tabla 5). El valor obtenido en la d de Cohen
(0.346) indicó un tamaño del efecto pequeño. El contraste de medias según el género no mostró diferencias significativas entre las puntuaciones en el test varones y mujeres.

Tabla 5. Contraste de Medias según curso

\begin{tabular}{cccccccc}
\hline Curso & $\mathbf{N}$ & Media & DT & t & gl & Sig. & d de Cohen \\
\hline $2^{\mathbf{0}}$ & 377 & 45.0818 & 15.4858 & -4.595 & 702.348 & 0.000 & 0.346 \\
$3^{0}$ & 328 & 50.1651 & 13.8872 & & & \\
\hline
\end{tabular}

El porcentaje promedio de respuestas correctas fue mayor en el tema de "Derechos y deberes" (Media: 68.55, SD: 25.85) y en el de "Identidad social y cultural" (Media: 60.74; SD: 29.88); dicho porcentaje fue inferior en los temas de "Historia de la cooperación europea" (Media: 51.43, SD: 29.67), "Ciudadanía europea" (Media: 48.49, SD: 21.72), "Europa en la vida cotidiana" (Media: 47.42, SD: 26.17), y "Las instituciones europeas" (Media: 22.16, SD: 20.90). Los resultados del ANOVA de medidas repetidas muestran diferencias significativas entre los porcentajes medios de respuestas correctas de los seis temas del test. El coeficiente $\eta^{2}$ parcial, 0.591 (Traza de Pillai: valor 0.591; F (5, 700): 202.106; p $<.000)$ sugiere un tamaño del efecto grande. Los resultados de las comparaciones múltiples muestran diferencias significativas entre los porcentajes medios de los seis temas del test, excepto en los casos de: Derechos humanos y responsabilidades/Identidad social y cultural, Europa en la vida cotidiana/ Ciudadanía europea, Europa en la vida cotidiana/Historia de la cooperación europea y Ciudadanía europea/Historia de la cooperación europea (Tabla 6). 
Tabla 6. Comparación por pares

\begin{tabular}{lcccc}
\hline \multicolumn{1}{c}{ (I) Tema } & \multirow{2}{*}{$(\mathbf{J})$ Tema } & $\begin{array}{c}\text { Diferencias de medias } \\
\text { I-J }\end{array}$ & Error Típico & Sig. \\
\hline & 2 & 2.931 & 1.437 & .626 \\
1. Derechos Humanos y & 3 & 16.236 & 1.428 & .000 \\
responsabilidades & 4 & 14.894 & 1.305 & .000 \\
& 5 & 12.719 & 1.528 & .000 \\
& 6 & 40.307 & 1.486 & .000 \\
\hline & 3 & 13.305 & 1,289 & .000 \\
2. Identidad social y cultural & 4 & 11.962 & 1.195 & .000 \\
& 5 & 9.787 & 1.381 & .000 \\
& 6 & 37.376 & 1.391 & .000 \\
\hline \multirow{2}{*}{ 3. Europa en la vida cotidiana } & 4 & -1.343 & 1.056 & 1.000 \\
& 5 & -3.518 & 1.272 & .087 \\
\hline \multirow{2}{*}{ 4. Ciudadanía europea } & 6 & 24.071 & 1.212 & .000 \\
\hline 5. Historia de la cooperación europea & 5 & -2.175 & 1.121 & .792 \\
\hline 6. Las instituciones europeas & 6 & 25.414 & 1.120 & .000 \\
\hline
\end{tabular}

Estos resultados muestran que el alumnado tiene más conocimiento sobre los temas de "Derechos humanos y responsabilidades" e "Identidad social y cultural", y un menor conocimiento sobre "La ciudadanía europea y la cooperación", "El papel de la UE en la vida cotidiana" y "Las instituciones europeas".

\section{Conclusiones}

La formación es la clave para la participación activa de los jóvenes en el proceso de integración europea. Los resultados de la investigación muestran que el alumnado tiene un nivel bajo-medio de conocimiento sobre el impacto de la UE en la vida cotidiana, la historia del proceso de cooperación europea, el ejercicio de los ciudadanos europeos y las instituciones de la UE, independientemente del año escolar, el sexo o la comunidad autónoma a la que pertenecen. Los resultados coinciden con los obtenidos en otros estudios (Horvath \& Paolini, 2013; Mahendran \& Cook, 2007) donde se observa que: a) los jóvenes saben menos sobre la UE que otros grupos de mayor edad, y b) exigen más información sobre las oportunidades que ofrece la adhesión de su país a la UE. Este estudio evidencia debilidades en el proceso de enseñanzaaprendizaje de la Educación para la Ciudadanía Europea (ECE) en los centros de
Enseñanza Secundaria en España. La ECE ha sido colocada en el centro del debate ideológico entre los principales partidos políticos en España. Los efectos de dicho debate se pueden ver en las dos últimas reformas educativas españolas, donde hay puntos de vista opuestos con respecto a los contenidos y la ubicación de la ECE en el plan de estudios de la Enseñanza Secundaria. Otros desafíos para la integración de la ECE en el currículo son el cronograma de asignaturas de Educación Secundaria, el excesivo academicismo del currículum y la falta de coordinación entre las asignaturas; tales circunstancias limitan la enseñanzaaprendizaje transversal de los contenidos de la ECE.

La desinformación conduce a la incomprensión, al distanciamiento y al bajo nivel de participación ciudadana (Hernández Rodríguez, 2008). El conocimiento limitado que muestra el alumando sobre temas relacionados con la integración y ciudadanía europea ilustra la necesidad de crear programas para mejorar la identidad europea de los jóvenes. Los adolescentes se encuentran en una etapa delicada en el proceso de crecimiento donde el problema de la identidad es palpable. La implementación de programas educativos para la ciudadanía ayuda al alumnado a crear y desarrollar sus 
múltiples identidades, es decir, a sentirse miembros y participantes de su entorno inmediato y distante (del Río, 2009).

Adquirir una conciencia cívica de los entornos en los que se desenvuelve nuestra vida es fundamental para ejercer una ciudadanía activa, participativa y efectiva en esos entornos. Según Jamieson (2007), Georgi (2008) y Verhaegen, Hooghe y Meeusen (2016), la educación para la ciudadanía europea debería basarse en el contacto con la UE y el aumento de las experiencias personales con la realidad europea. Los intercambios educativos entre alumnado $\mathrm{y}$ profesorado de los Estados miembros son esenciales para generar el sentimiento de pertenencia a la UE.

El análisis del nivel de conocimiento del alumnado español sobre la ciudadanía europea ha sido el punto de partida para el diseño, desarrollo e implementación del programa ECLIPSE (Bombardelli \& Santana, 2014). Este programa de formación, diseñado para ser integrado en las áreas curriculares de la Educación Secundaria, tiene como objetivo promover: 1) la construcción de la identidad del alumnado como jóvenes ciudadanos europeos; 2) desarrollar competencias clave tales como aprender a aprender, la competencia de la autonomía e iniciativa personal y la competencia cívica; 3) promover la dimensión europea en la educación en respuesta a los desafíos de la Estrategia Europa 2020. ECLIPSE es un programa común a nivel europeo y como tal presenta un valor agregado; amplía los horizontes al permitir al alumnado estar más en contacto con la vida en los Estados miembros, al descubrir que los países europeos tienen mucho en común, así como diferencias.

Fischman y Haas (2012, p.174) señalan que la consolidación de cualquier identidad dada, ya sea "personal", "nacional" o "comunitaria", es siempre un proyecto "educativo" inconcluso. El énfasis que el programa ECLIPSE otorga a la ciudadanía europea "competente" debe alentar al alumnado a saber cómo quieren que sea el desarrollo de Europa, y a participar en el mismo, percibiéndose como responsables a nivel local, nacional, europeo e internacional, compartiendo un destino común.

La cooperación de los estudiantes con jóvenes de otros países europeos debería alentarlos a mirar más de cerca la vida cotidiana de sus iguales; familiarizándose con ellos a través de las redes sociales, lo que ayudaría a superar posibles prejuicios y aumentar su deseo de conocerse mejor. Trabajando juntos a través de las fronteras, el alumnado y el profesorado se conocerán entre sí, aprenderán sobre las diferentes instituciones y países, sobre las formas de trabajo, y practicarán la cooperación intercultural. Se espera que esto tenga un impacto positivo en la motivación para desarrollar el sentimiento de ciudadanía europea.

Las escuelas deberían dar al alumnado la oportunidad de experimentar activamente la ciudadanía y ofrecerles la posibilidad de estudiar y discutir el significado de ser un ciudadano europeo. En realidad, no podemos enseñar al alumnado a ser ciudadanos; solo podemos generar entornos de aprendizaje apropiados para aprender a ser ciudadanos críticos de una UE en construcción.

La formación para la ciudadanía es un proceso complejo y multifacético integrado en la cultura y los sistemas educativos de las democracias de Europa, que requiere la exploración de nuevos modelos educativos en muchos países (Torney-Purta, 2002). El destino de la UE reside en una generación de jóvenes que se mueven entre la atracción de los beneficios de la integración europea y el sentimiento de ser ignorados por las instituciones políticas que están distanciadas de sus necesidades e intereses (EACEA, 2013; Sloam, 2013 Tsafos, 2006;). Los resultados de nuestra investigación muestran que algunos temas sobre la ciudadanía europea necesitan un mayor énfasis en el plan de estudios de Educación Secundaria para que los alumnos puedan: a) mejorar su conocimiento de la UE; b) ganar experiencias participativas y c) tomar conciencia del significado de ser ciudadanos europeos tanto 
en la UE como en el mundo. El euroescepticismo ha crecido en los Estados miembros de la UE, cuestionando el modelo europeo de ciudadanos que se está construyendo. Esta circunstancia requiere un debate profundo entre los jóvenes para analizar críticamente las causas del aumento de la eurofobia (Comisión Europea, 2015). Es necesario desarrollar en el alumnado la voluntad de participar en la construcción de la UE.

El proceso de integración de la UE y la ciudadanía europea deben ser incluidos en el plan de estudios de la Educación Secundaria, y estar sujetos a la reflexión individual y grupal en el aula. El programa ECLIPSE propone un modelo alternativo de educación para la ciudadanía apoyado en contenidos transversales, $y$ es una alternativa al academicismo imperante en las asignaturas de secundaria. En este modelo, el docente asume el reto de adaptar y/o diseñar actividades en colaboración con otros profesores, b) actuar como catalizador de las sesiones de clase, y c) trabajar temas de ciudadanía europea en las diferentes asignaturas del plan de estudios de manera coordinada.

El bajo número de sujetos que participan en el estudio limita la generalización de los resultados. Se debería realizar nuevas investigaciones con muestras más grandes que incluya alumnado de otras Comunidades Autónomas para confirmar el alcance de las limitaciones del conocimiento sobre la UE. El estudio solo aborda cuantitativamente el nivel de conocimiento sobre la UE. Este análisis cuantitativo debe combinarse con una investigación cualitativa para estudiar con mayor profundidad la génesis de los errores o tergiversaciones del alumnado sobre el proceso de integración de la UE y la construcción de la ciudadanía europea. Además, es necesario diseñar una evaluación de la competencia ciudadana, no solo del conocimiento sobre la UE (Torney-Purta, Cabrera, Crotts, Liu, y Rios, 2015).

La investigación abre nuevas vías de estudio sobre el conocimiento sobre la UE y la ciudadanía europea. Sería aconsejable abordar las siguientes preguntas desde una perspectiva cuantitativa y cualitativa: ¿existen diferencias en el conocimiento sobre la UE entre el alumnado de Educación Secundaria Obligatoria, Educación Secundaria no Obligatoria y Universidad? ¿Hay diferencias en el conocimiento del alumnado sobre la UE según su situación socioeconómica y Comunidad Autónoma de origen? ¿Qué interés tiene el alumnado en obtener información sobre la UE para su futuro personal $\mathrm{y}$ profesional? ¿Existe relación entre el nivel de información sobre la UE y las actitudes hacia el proceso de integración europea y la ciudadanía europea? ¿El grado de conocimiento de los alumnos sobre la UE afecta a la forma de argumentar las ventajas y limitaciones de la pertenencia a la UE? ¿El nivel de conocimiento de los alumnos sobre la UE afecta sus intenciones de ejercer una ciudadanía activa? ¿En qué medida están presentes los valores de la UE en los proyectos de vida de los alumnos? ¿El conocimiento del alumnado sobre las oportunidades que ofrece la ciudadanía europea afecta a la forma en que priorizan sus objetivos de vida?

\section{Referencias}

Agidarg, O., Huyst, P. \& Van Houte, M. (2012). Determinants of the formation of a European identity among children: individual and school level influence. Journal of Common Market Studies, 50, 198-213. doi: http://doi.org/10.1111/j.14685965.2011.02205.X

Amadeo, J., Torney-Purta, J., Lehmann, R., Husfeldt, V. \& Nikolova, R. (2002). Civic knowledge and engagement among upper secondary pupils in sixteen countries. Amsterdam: IEA.

Bombardelli, O. \& Santana, L.E. (2014). European citizens are groving up. Madrid: EOS.

Bovero, M. (2002). Una gramática de la democracia: contra el gobierno de los peores. Madrid: Trotta.

Committee on Culture and Education of European Parliament (2016). On Learning 
EU at school (2015/2138(INI), Retrieved from

http://www.europarl.europa.eu/sides/getDoc. do?pubRef=-//EP//TEXT+REPORT+A82016-0021+0+DOC+XML+V0//EN

Council of the European Union (2014). Council Regulation (EU) No 390/2014 of 14 April 2014 establishing the 'Europe for Citizens' programme for the period 20142020. Official Journal of the European Union, 17/4/14. 1.115/3

del Río, S. (2009). Reactivar la ciudadanía europea comunicando Europa. Pliegos de Yuste. Revista de Cultura, Ciencia y Pensamiento Europeo, 9-10, 67-76.

EACEA (2012). Citizenship education at schools in Europe. Brussels: EACEA.

EACEA (2013). Youth participation in democratic life. Brussels: EACEA.

EACEA (2016). Promoting citizenship and the common values of freedom, tolerance and non-discrimination through education. Brussels: EACEA.

Ebel, R.L., \& Frisbie, D.A. (1986). Essentials of education measurement. Englewood Cliffs, NJ: Prentice Hall.

European Commission (2011). European Commission proposes to make 2013 "The European Year of Citizens". Retrieved from http://europa.eu/rapid/press-release_IP-11959_en.htm

European Commission (2012). European citizenship. Standard Eurobarometer 77. Brussels: European Commission.

European Commission (2013). European Union Citizenship Flash Eurobarometer 365. Brussels: European Commission.

European Commission (2014). European Citizenship Standard Eurobarometer 81. Brussels: European Commission.

European Commission (2015). ANNEX to the Commission implementing decision on the adoption of the 2016 work programme and the financing for the implementation of the Europe for Citizens programme. Brussels: European Commission.
EURYDICE (2005). La Educación para la ciudadanía en el contexto escolar europeo, Bruselas: EURYDICE.

Fischman, G \& Haas, E. (2012). Beyond idealized citizenship education: Embodied cognition, metaphors, and democracy. Review of Research in Education, 36, 169196.

doi:

http://doi.org/10.3102/0091732X11420927

Georgi, V.B. (2008). Citizens in the Making: Youth and Citizenship Education in Europe. Child Development Perspectives, 2, 107113. doi: http://doi.org/10.1111/j.17508606.2008.00050.x

Greppi, A. (2012). La democracia y su contrario. Representación, separación de poderes y opinión pública. Madrid: Trotta.

Halász, G. \& Michel, A. (2011). Key competences in Europe: interpretation, policy formulation and implementation. European Journal of Education, 46, 289306. doi: http://doi.org/10.1111/j.14653435.2011.01491.x

Hernández Rodríguez, E. (2008). Acercar Europa a los europeos: un reto vital. Madrid: Euroeditions, Books for Europe's future.

Horvath, A. \& Paolini, G. (2013). Political participation and EU citizenship: Perceptions and behaviours of young people. Brussels: EACEA.

Huyst, P. (2009). The Europeans of tomorrow: Researching European identity among young Europeans. Gent University, Centre for EU Studies.

Jamieson, L. (Coord.) (2007). Orientations of young men and women to citizenship and European identity. Brussels: European Commission. Directorate General for Research Citizen and Governance in a knowledge-based society.

Kerr, D., Sturman, L., Schulz, W. \& Burge, B. (2010). ICCS 2009 European Report. Civic knowledge, attitudes and engagement among lower secondary pupils in 24 European countries. Amsterdam: 
International Association for the Evaluation of Educational Achievement.

Kuhn, T. (2016). The social stratification of European schoolchildren's transnational experiences: A cross-country analysis of the International Civics and Citizenship Study. European Sociological Review, 32(2), 266279. doi: http://doi.org/10.1093/esr/jcv097

Mahendran, K. \& Cook, D. (2007). Young people's views on participation and their attitudes towards the European Union: building a bridge between Europe and its citizens. Edinburg: Scottish Executive Social Research.

Marshall, H. (2009). Educating the European citizen in the global age: engaging with the post-national and identifying a research agenda. Journal of Curriculum Studies, 41, 247-267. doi: http://doi.org/10.1080/00220270802642002

Milana, M. (2008). Is the European (active) citizenship ideal fostering inclusion within the Union? A critical review. European Journal of Education, 43, 207-216. doi: http://doi.org/10.1111/j.1465-

3435.2008.00344.x

Myers, J.P. \& Zaman, H.A. (2009). Negotiating the global and national: Immigrant and dominant culture adolescents' vocabularies of citizenship in a transnational world. Teachers College Record, 111(11), 2589-2625.

Morales, P. (2012). Análisis de ítems en las pruebas objetivas. Madrid: Universidad Pontificia Comillas, Facultad de Ciencias Humanas y Sociales.

Naval, C., Print, M. \& Veldhuis, R. (2002). Education for democratic citizenship in the new Europe: Context and reform. European Journal of Education, 37, 107-128. doi: http://doi.org/10.1111/1467-3435

Sloam, J. (2013). Voice and equality. Young people's politics in the European Union. West European Politics, 36, 1-23. doi: http://doi.org/10.1080/01402382.2012.749652
Torney-Purta, J. (2002). Patterns in the civic knowledge. Engagement. and attitudes of European adolescents: The IEA Civic Education Study. European Journal of Education, 37, 129-141. doi: http://doi.org/10.1111/1467-3435.00098

Torney-Purta, J., Cabrera, J., Crotts, K., Liu, O.L. \& Rios, J. (2015). Assessing Civic Competency and Engagement in Higher Education: Research Background, Frameworks, and Directions for NextGeneration Assessment. ETS Research Report Series, 2, 1-48. doi: http://doi.org/10.1002/ets2.12081

Tsafos, N. (2006). Growing up a European. SAIS Review of International Affairs, 26, p. 181. doi: https://doi.org/10.1353/sais.2006.0046

UNESCO (2015). Global Citizenship Education. Topics and learning objectives. Paris: United Nations Educational, Scientific and Cultural Organization.

Verhaegen, S., Hooghe, M. y Meeusen, C. (2016). Opportunities to learn about Europe at school. A comparative analysis among European adolescents in 21 European member states. Journal of Curriculum Studies, 45(6), 838-864. doi: http://doi.org/10.1080/00220272.2013.8009 $\underline{95}$

\section{AGRADECIMIENTOS}

El proyecto ECLIPSE ha sido cofinanciado por la Agencia Ejecutiva en el ámbito Educativo, Audiovisual y Cultural (EACEA), número de referencia 517815-LLP-1-2011-1IT COMENIUS-CMP.

Este artículo refleja las opiniones únicamente de los autores, la Comisión Europea no se hace responsable del uso que pueda hacerse de la información contenida en el mismo. 


\section{Authors / Autores}

Santana-Vega, Lidia E. (lsantana@ull.es)

Catedrática de Universidad en el área de Didáctica y Organización Escolar de la Universidad de La Laguna (España). Coordinadora del Grupo de Investigación en Orientación Educativa y Sociolaboral (GIOES). Sus áreas de investigación están relacionadas con la educación secundaria, la educación para la ciudadanía, la evaluación educativa, las diferencias de género, y la orientación para la carrera. Su dirección postal es: Universidad de La Laguna - Facultad de Educación. A.C. 456 - 38200 San Cristóbal de La Laguna. S/C de Tenerife (España).

\section{Feliciano-García, Luis ( lfelici@ull.edu.es)}

Titular de Universidad en el área de Métodos de Investigación y Diagnóstico en Educación de la Universidad de La Laguna (España). Miembro del Grupo de Investigación en Orientación Educativa y Sociolaboral (GIOES). Sus áreas de investigación están relacionadas con educación secundaria, educación para la ciudadanía, evaluación educativa, diferencias de género, orientación para la carrera. Su dirección postal es Su dirección postal es: Universidad de La Laguna Facultad de Educación. A.C. 456 - 38200 San Cristóbal de La Laguna. S/C de Tenerife (España).

\section{RELIEVE}

\section{Revista ELectrónica de Investigación y EValuación Educativa E-Journal of Educational Research, Assessment and Evaluation}

[ISSN: 1134-4032]

(C) Copyright, RELIEVE. Reproduction and distribution of this articles it is authorized if the content is no modified and their origin is indicated (RELIEVE Journal, volume, number and electronic address of the document).

(C) Copyright, RELIEVE. Se autoriza la reproducción y distribución de este artículo siempre que no se modifique el contenido y se indique su origen (RELIEVE, volumen, número y dirección electrónica del documento). 